\title{
IceCube results
}

\section{Klas Hultqvist ${ }^{* \dagger}$}

Oskar Klein Centre and Department of Physics, Stockholm University

E-mail: klas.hultqvistefysik.su.se

The IceCube Neutrino Observatory started taking data in its full configuration in 2011. A diffuse astrophysical flux of neutrinos has been detected. Although the searches for point sources, steady or transient, have not yet yielded positive results they do have implications for the origin of the diffuse flux. This contribution summarises IceCube results on astrophysical neutrino and cosmic ray fluxes.

XVI International Workshop on Neutrino Telescopes,

2-6 March 2015

Palazzo Franchetti - Istituto Veneto, Venice, Italy

\footnotetext{
*Speaker.

${ }^{\dagger}$ For the IceCube Collaboration.
} 


\section{Introduction}

For about forty years, efforts have been under way to detect interactions of astrophysical high energy neutrinos by means of Cherenkov light in natural media[1]. As of two years ago, this has become reality. The focus is now shifting towards characterization and interpretation of the observed neutrino signal.

The interest in neutrinos as cosmic messengers stems from their ability to reach us over cosmic distances, directly from their point of creation, unaffected by matter, magnetic fields or background radiation. They are produced in interactions of cosmic ray particles, and possible in hitherto unknown processes, and could carry unique information about processes the universe.

The IceCube Neutrino Observatory at the South Pole consists of 86 vertical strings of photosensors (Digital Optical Modules, DOMs), deployed at depths between 1450m and 2450m in the polar ice cap (IceCube), and an air shower array covering the ice surface immediately above (IceTop). The string spacing is $125 \mathrm{~m}$, and the distance between DOMs on a string is $17 \mathrm{~m}$. The central lower part of the detector, IceCube DeepCore, has a denser instrumentation in order to enhance the sensitivity to lower energy events. The DOMs detect the Cherenkov light emitted by relativistic charged particles traversing the ice. During construction, data were taken with 9, 22, 40, 59, and 79 strings. These samples are referred to as IC9-IC79. The IC79 configuration, from 2010, is very similar to the final one (IC86).

The two most important event topologies are tracks and cascades. Tracks, created by muons from $v_{\mu}$ charged current interactions ${ }^{1}$ give good angular resolution because of the lever arm provided (the angle between the incoming neutrino and the muon is small at $\mathrm{TeV}$ energies), but only very indirect information on energy of the incoming neutrino, since the muon track will usually just cross the detector. Particle showers (cascades) created in neutrino interactions have a small spatial extent in comparison to the detector array. The lack of a lever arm means the directional reconstruction is rather poor $\left(\gtrsim 15^{\circ}\right)$, but the deposited energy is a much better proxy for the neutrino energy than in the case of tracks. At high energy, where the $\tau$ decay length is significant, $v_{\tau}$ interactions can give several topologies involving tracks and cascades[2, 3]. These have not been observed yet.

The highlight of IceCube physics so far is the discovery of a diffuse astrophysical neutrino flux. Localised point sources have not been detected yet, but the negative results have implications for the origin of the diffuse flux. IceCube addresses a wide range of other topics, including cosmic ray and atmospheric neutrino physics, supernova neutrino emission, searches for dark matter and fundamental properties of space-time. One interesting development is the successful use of DeepCore to measure neutrino oscillations at higher energy than before. This has led to the plans for PINGU[4] which is presented elsewhere in these proceedings. This contribution summarises some IceCube measurements in the fields of charged cosmic rays and astrophysical neutrinos.

\section{Cosmic Rays}

Charged cosmic rays are central to the physics of neutrino telescopes. Identifying their sources through neutrino emission is a fundamental aim of neutrino astronomy. Their interactions in the atmosphere create extensive air showers giving rise to a large background of downgoing muon

\footnotetext{
${ }^{1}$ No distinction between neutrinos and antineutrinos will be made here.
} 


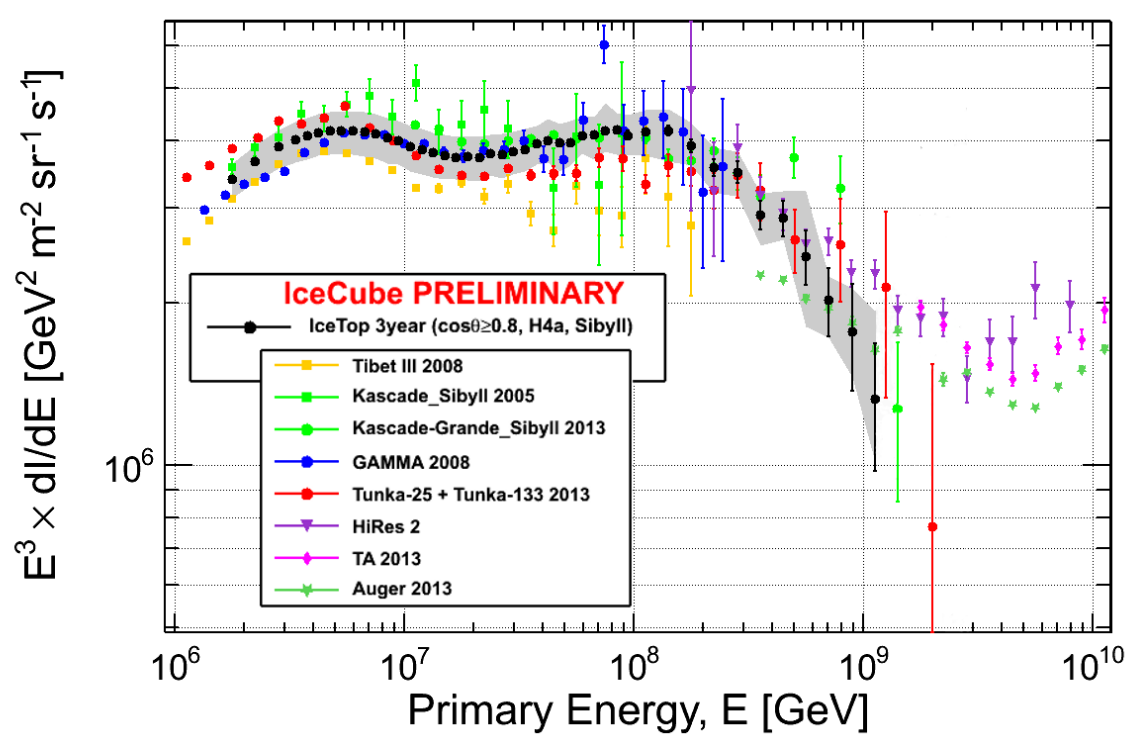

Figure 1: Cosmic ray flux reconstructed using three years of data for 73 IceTop stations.

events, and a rather isotropic flux of neutrinos. This flux is an opportunity to study the properties of neutrinos, but it is also a background to astrophysical neutrino signals, and the extraction of these will benefit from an accurate model for atmospheric production. Also, the highest energy cosmic ray collisions occur at energies which cannot be probed in other ways, and thus have an intrinsic particle physics interest.

IceCube is able to detect cosmic ray particle showers at the ice surface, close to the shower maximum for the highest primary energies, and in the deep ice, where the muonic component of the shower can be studied.

The IceTop surface array comprises 81 stations, each consisting of two ice-filled tanks viewed by DOMs. With IceTop, the lateral shower profile can be reconstructed. For a given type of cosmic ray primary, or primary composition, the particle density $125 \mathrm{~m}$ from the shower core is used as a proxy for the primary energy. Assuming the composition of the H4a model [5], the spectrum presented in Fig. 1, updated from Ref. 6 with another two years of data, was derived.

The threshold in terms of primary cosmic ray energy is lower for the in-ice detector than for IceTop. A sample of $2.5 \times 10^{11}$ cosmic ray muon events has been used to map the variation of cosmic ray intensity with right ascension for different declinations [7], as shown in Fig. 2. An angular smoothing with a radius of $20^{\circ}$ has been applied to these maps in order to show the largescale structure. (Subtracting the dipole and quadropole components, reveals a finer structure which could reflect the configuration of the local interstellar magnetic field[8].) The dipole-like structure in the figure is thought to arise from diffusion in the galactic magnetic field of cosmic rays from several sources[9]. At around $100 \mathrm{TeV}$ the direction of the maximum changes, from the Orion arm of the galaxy towards the galactic centre.

\section{Starting events}

Traditionally, for neutrino telescopes, muon track events have been in focus. The reason is that these give a well-reconstructed direction which can be used both to look for astrophysical point 

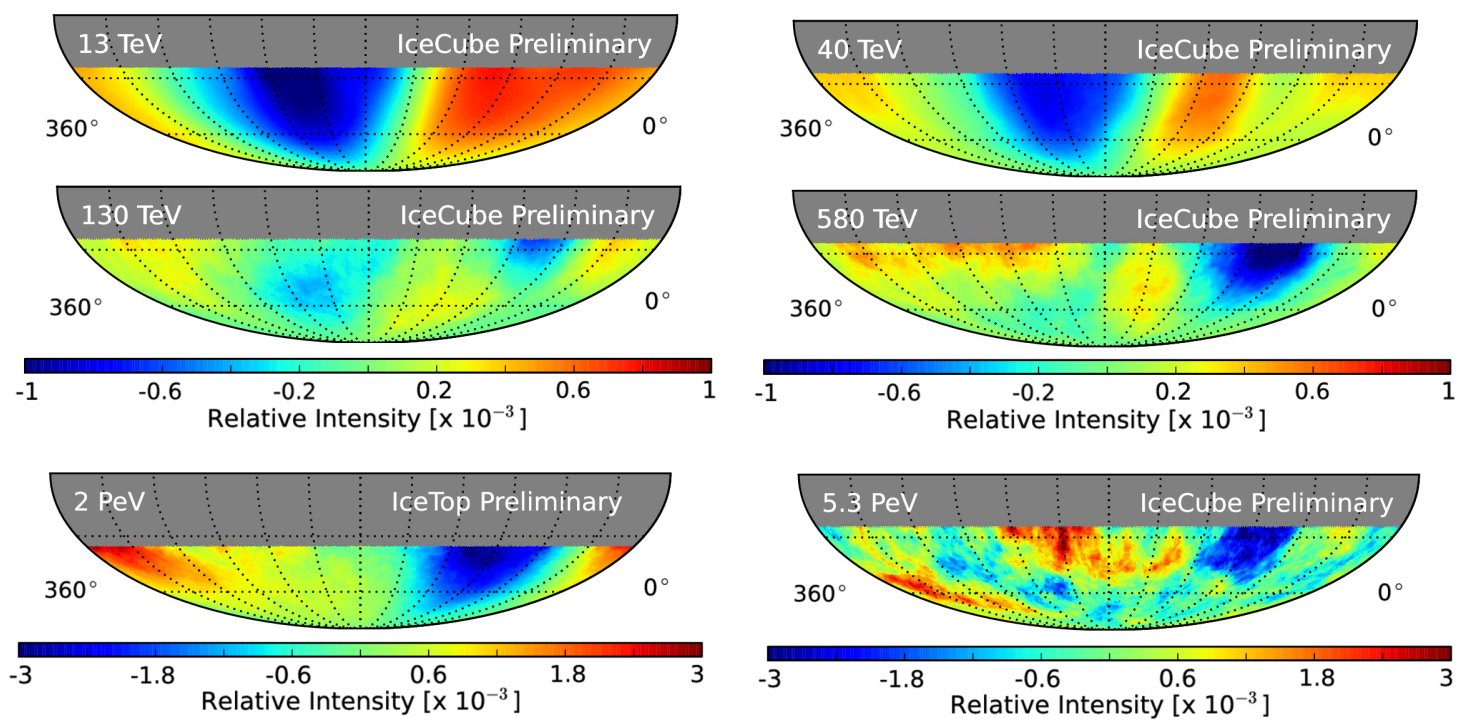

Figure 2: Large-scale relative cosmic ray intensity maps in equatorial coordinates for selections with different median primary energy. The bottom left plot was obtained using IceTop, with a requirement of at least eight hit stations.

sources and to select upward-going events, thereby using the earth as a filter against atmospheric muon background. For upgoing events, the effective volume is large since the muon can reach the instrumented volume from $v_{\mu}$ interactions far outside.

However, it was the study of "starting events" that revealed the astrophysical neutrino flux. This selection method was introduced in order to search for additional events, following the chance observation of two $\sim \mathrm{PeV}$ cascade events in a search for much higher energy neutrinos from the GZK process [10]. Neutrino interactions inside IceCube are selected by the requirement that the visible event starts to develop well inside the instrumented volume, without any sign of incoming particles. The outer layers of DOMs are thus used as a veto against incoming events, which means that atmospheric muons can be rejected. Also downgoing atmospheric neutrinos can be removed by the veto, since they will often have accompanying muons from the same cosmic ray shower [11].

The high energy starting events (HESE) search uses simple criteria: The total charge collected by the DOMs must exceed 6000 photoelectrons (PE), and out of the first $250 \mathrm{PE}$ deposited at most 2 PE may be in a veto region defined close to the detector boundary. The first such analysis [12] selected 28 events in two years of data (IC79 and IC86). Adding a third year [13] increased this number to 37 events, with an expected background of 15 events $(8.4 \pm 4.2$ atmospheric muon events and 6.6 ${ }^{+5.9}$ 1.6 atmospheric neutrinos, including prompt neutrinos from the decay of heavy quarks). Accounting for the different energy and zenith angle distributions, as compared to background, this established the astrophysical flux at 5.7 $\sigma$. A fourth year of data has now been added, increasing the significance to $\sim 7 \sigma$. Fig. 3 shows the observed values of declination and deposited energy of the 54 observed events.

The analysis selects both cascade events and tracks. However, most neutrino interactions give cascades; only $v_{\mu}$ charged crurent interactions predominantly give track-like events. Furthermore, for muon track events, energy is carried out of the instrumented volume by the muon. This means 


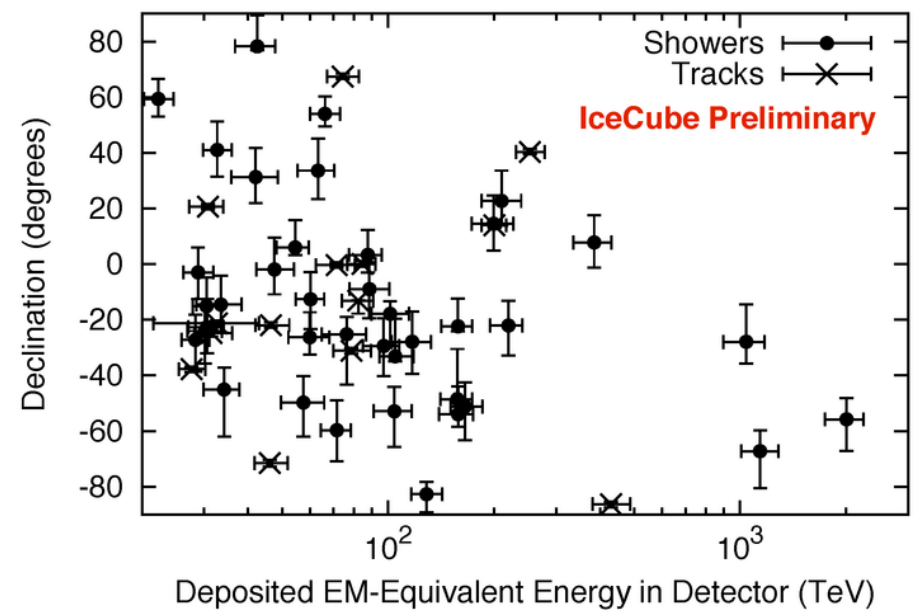

Figure 3: Declination and deposited energy of observed high energy starting events in four years of IceCube data.
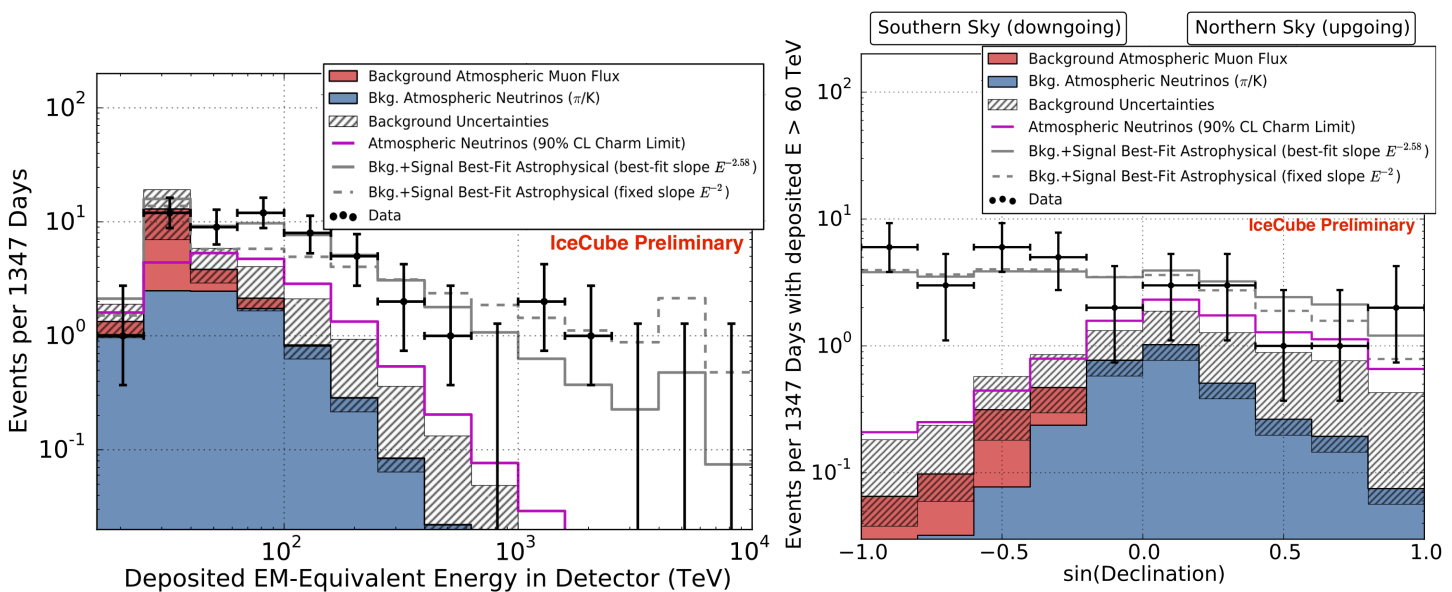

Figure 4: Deposited energy and sine of the declination for events in the HESE sample. The grey lines are for fits to the data, including an isotropic astrophysical flux. The solid line is for the best-fit spectral index (2.58); the dashed line is for a fit with the index fixed at 2 .

that most signal events detected will be cascades. In addition, some of the numerous background muons of moderate energy are dim enough to escape the veto, and if they suffer a catastrophic energy loss they can create a shower which passes the charge cut. Therefore, the sample is dominated by cascades (shower-like events), as can be seen from the figure. In the upper right part of Fig. 3, there is a depletion caused by absorption of high-energy neutrinos in the earth.

Fig. 4 (left) shows the energy distribution of the selected events, and expected backgrounds. The declination distribution to the right is for events that have a deposited energy greater than $60 \mathrm{TeV}$. This sample was used for a fit based on a joint likelihood for the two variables in Fig. 4. The signal dominates in the downgoing and high energy region. The atmospheric neutrino background from $\pi$ and $\mathrm{K}$ decays (the conventional flux) peaks close to the horizon, and is suppressed by the veto for downgoing events. The prompt atmospheric flux (predominantly from charm) is mostly 


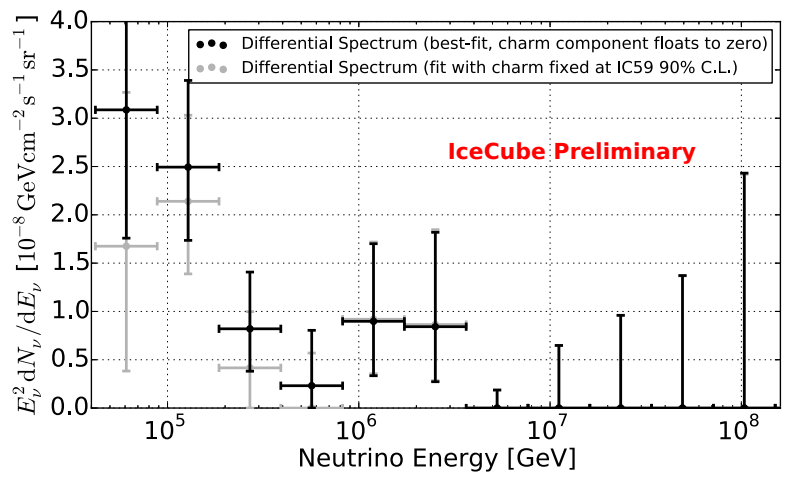

Figure 5: Unfolded astrophysical energy spectrum obtained by fitting to four years of HESE data. The grey points show the effect of imposing a large prompt component in the fit.

isotropic, but is also suppressed by the veto and, because its of higher energy, by Earth absoption.

There were three PeV events in the first three years, but none in the fourth year. Consequently the best fit power-law spectrum has softened from a spectral index of $\gamma=2.3 \pm 0.3$ with three years of data to $\gamma=2.6$ with four years, steeper than the $\gamma \sim 2$ expected for Fermi shock acceleration. Assuming equal fluxes of the three flavours, and an $E^{-2}$ spectrum, the best-fit flux per flavour at $100 \mathrm{TeV}$ for the the four-year analysis is $(0.84 \pm 0.30) \times 10^{-18} \mathrm{GeV}^{-1} \mathrm{~cm}^{-2} \mathrm{~s}^{-1} \mathrm{sr}^{-1}$.

For a more model independent presentation, Fig. 5 shows an unfolded astrophysical neutrino energy spectrum. For each point, the spectrum is assumed to have an $E^{-2}$ spectrum in the interval indicated by the horizontal error bar. The fit has been extended to include the normalisations in the different energy intervals as independent parameters. (Thus, the unfolded spectrum is a linear combination of basis functions which are nonzero in non-overlapping intervals of true neutrino energy.)

The efficiency of the simple veto of the HESE search falls with decreasing energy of the entering muons. This sets the lower energy threshold for the HESE search. To look for lower energy starting events, more DOMs have to be used in the veto. This will increase the number of random coincidences, giving false vetoes, unless additional geometrical causality criteria are imposed. Such an analysis has been performed, using two years of IceCube data [14]. The resulting distributions of deposited energy for events in the southern and northern sky, respectively, are shown in figure 6. Assuming equal fluxes of the three neutrino flavours, the best-fit all-flavour power-law spectrum from this analysis is

$$
\Phi_{v}=\Phi_{0}\left(\frac{E}{100 \mathrm{TeV}}\right)^{\gamma}=6.2_{-0.8}^{+1.1} \cdot 10^{-18}\left(\frac{E_{V}}{100 \mathrm{TeV}}\right)^{-2.46 \pm 0.12} \mathrm{GeV}^{-1} \mathrm{~cm}^{-2} \mathrm{sr}^{-1} \mathrm{~s}^{-1} .
$$

\section{Upgoing muons}

Upgoing muon events provide a high statistics sample with good pointing resolution, and low atmospheric muon background. The astrophysical component can identified, against the background of atmospheric neutrinos, by its harder energy spectrum. However, the observed energy is only very indirectly related to the neutrino energy. Fig. 7 shows the distribution of an energy proxy for an analysis based on two years of data (IC79 and IC86 configurations). The significance for an 


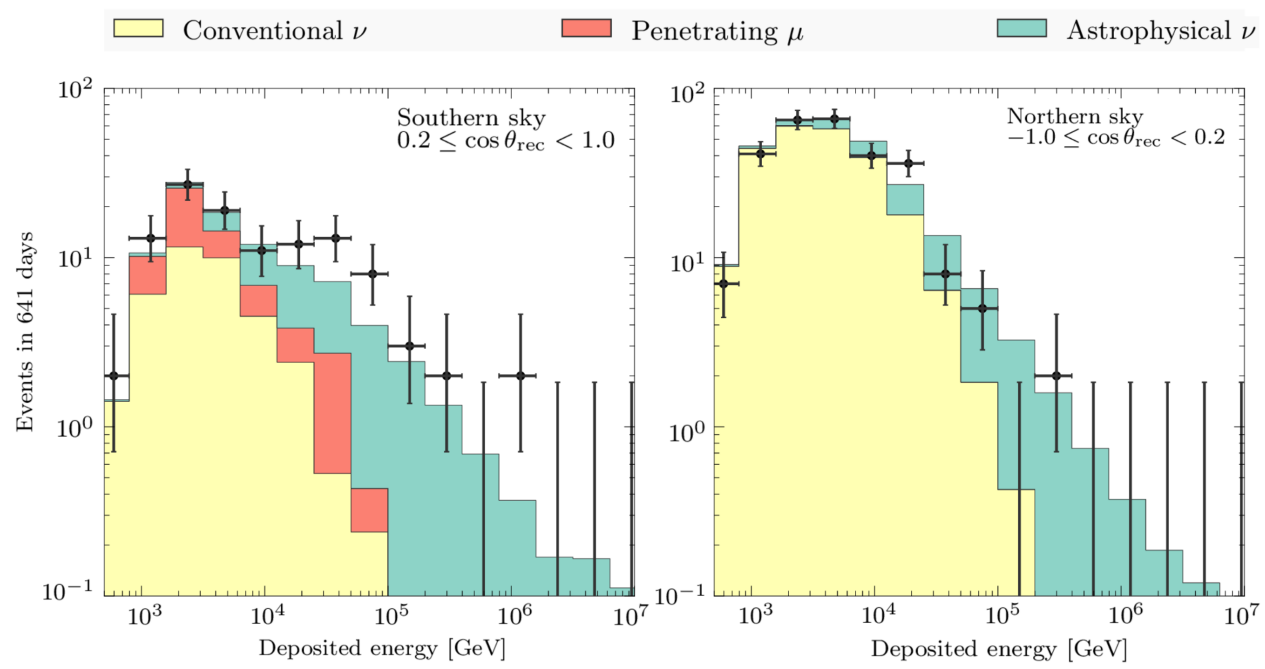

Figure 6: Deposited energy for the starting event search extending down to $1 \mathrm{TeV}$ in deposited energy.

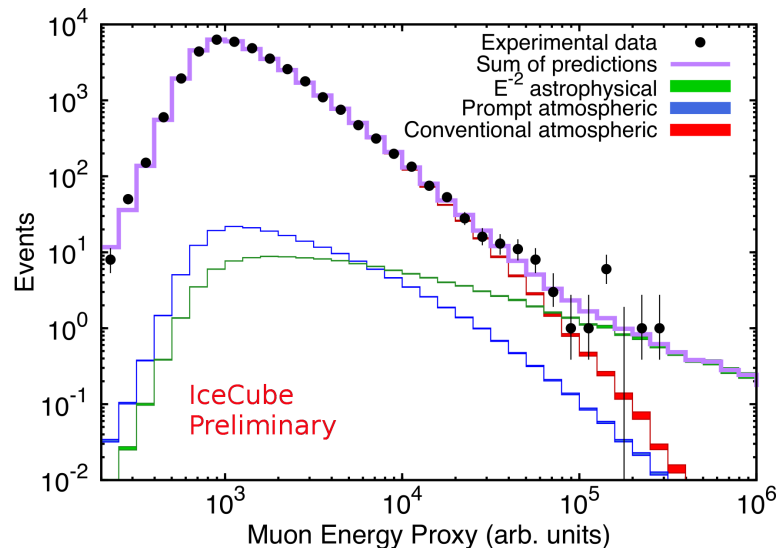

Figure 7: Distribution of energy proxy for upgoing muon events in two years of IceCube data.

extraterrestrial flux is $3.7 \sigma$, lending important independent support to the observations of starting events. The best-fit power law index is $2.2 \pm 0.2$, somewhat harder than that obtained for the starting events. Fig. 8 shows an unfolded $v_{\mu}$ energy spectrum for the IC79 data. Here, a spectrum is fitted to the data as a linear combination of orthogonal, but overlapping, spline functions [15]. It is then integrated to give the bin contents shown in the figure.

\section{Combined results}

By combining data selected with different methods, and for different time periods, the astrophysical flux can be constrained further. One example of this is the flavour composition. For production in pion decay, the composition at Earth is expected to be close to $\left(v_{e}: v_{\mu}: v_{\tau}\right)_{\oplus}=(1$ : $1: 1)$ [2], which is consistent with the observed proportions between tracks and cascades in the HESE sample. Other composition at the source will give moderate deviations from $(1: 1: 1)$ [16]. Non-standard neutrino properties or propagation effects could cause larger asymmetries at Earth. 


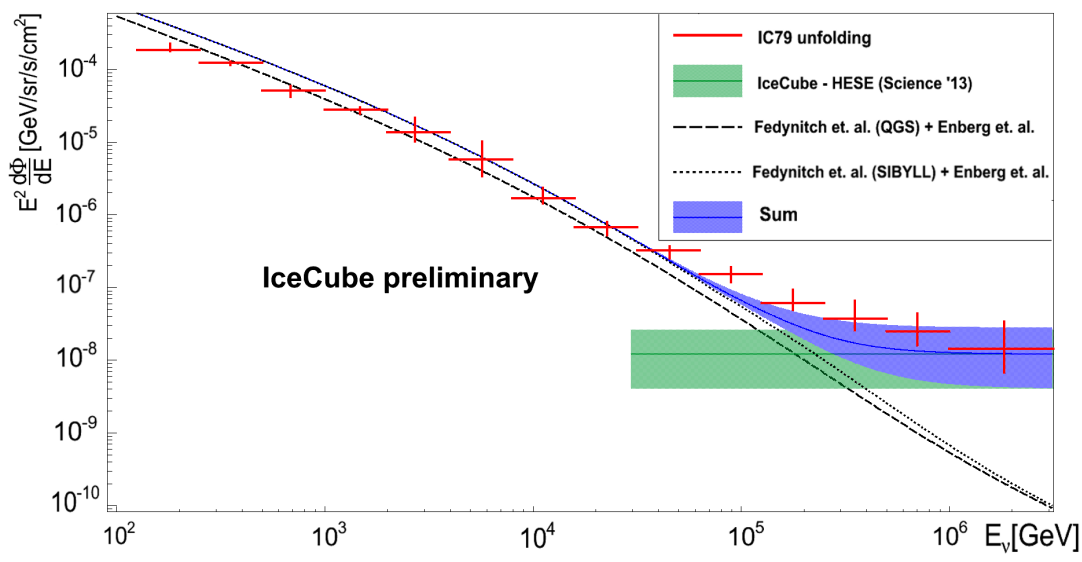

Figure 8: Unfolded muon neutrino energy spectrum from upgoing muons in the IC79 configuration. The blue band indicates the sum of expectations, including the astrophysical $E^{-2}$ flux fitted to the HESE data.

By combining the HESE track events with a lower energy veto-based cascade selection [17], limits were set which exclude $v_{\mu}$ only and $v_{e}$ only at $3.3 \sigma$ and $2.3 \sigma$, respectively (see grey contours in Fig. 9).

A more ambitious combination of data is a global fit to several diffuse analysis samples spanning five years and detector configurations from 40 to 86 strings. Six event samples were combined, including the two starting events selections ${ }^{2}$ and the two-year upgoing muon sample discussed above. Additional requirements were introduced to ensure that the samples are statistically independent. Fig. 9 shows the result of a fit allowing different fluxes for different neutrino flavours. Pure $v_{e}$ production at the source, which would be indicative of neutron decay, is excluded at $3.6 \sigma$. Fig. 10 shows energy spectra obtained in the global analysis.

For this analysis, the best-fit all-flavour power-law spectrum parameters in equation 1 are $\gamma=2.50 \pm 0.09$ and $\Phi_{0}=6.7_{-1.2}^{+1.1} \cdot 10^{-18} \mathrm{GeV}^{-1} \mathrm{~cm}^{-2} \mathrm{~s}^{-1} \mathrm{sr}^{-1}$.

\section{Sources}

The interpretation of the discovered flux is not clear. There are many potential sources of astrophysical neutrino emission; prominent candidates are active galactic nuclei, gamma-ray bursts, starburst galaxies, microquasars, supernova remnants, and pulsar wind nebulae [18]. The observed flavour composition is consistent with the general picture of production in decays of pions and kaons from interactions of accelerated hadrons. If the somewhat harder astrophysical flux fitted to the upgoing muon sample is a real effect, it could indicate several populations of sources. Fits to other spectral shapes than a power law have been performed, but at present no evidence for a different shape has been found. More statistics, and a systematic analysis aimed at finding a better description than a power law, should provide additional constraints on the mechanisms at work. This also includes studying different regions in the sky, the galactic plane in particular.

Searches for point sources, steady or transient, and extended sources, is a very active field

\footnotetext{
${ }^{2}$ The fourth year HESE sample was not included.
} 


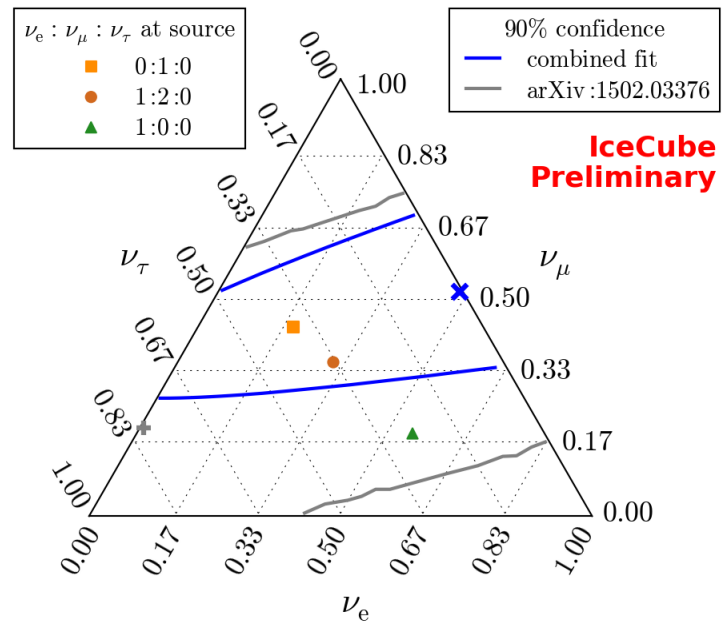

Figure 9: Result of a fit assuming identical spectral shapes, but different normalisation, of the three neutrino flavours at Earth. Blue shows the result of the global fit, whereas grey indicates the results from Ref. 17.

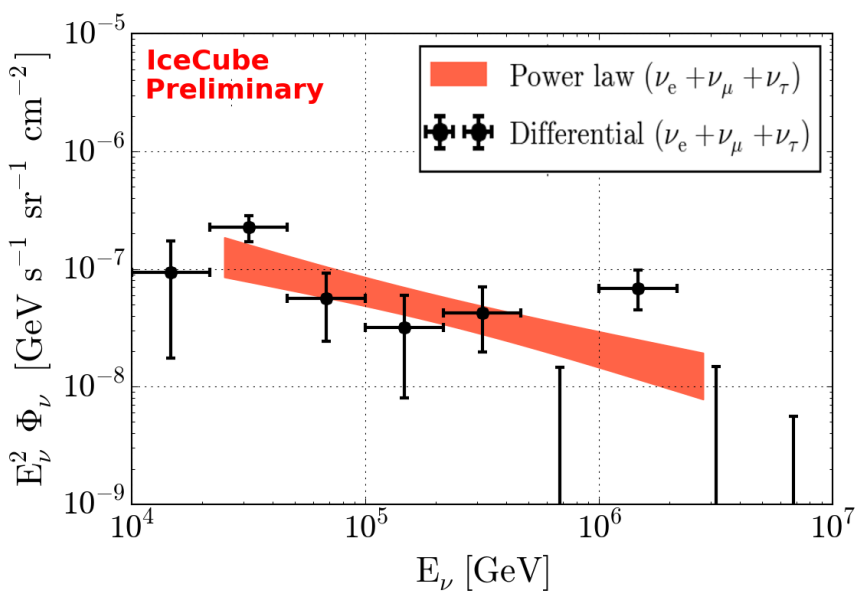

Figure 10: Best fit power-law spectrum (coloured band) and unfolded step-wise $E^{-2}$ spectrum (points).

of analysis in IceCube. None have been found ${ }^{3}$, although the detected flux implies that several hundred astrophysical track events per year are included in the experimental data. The sensitivity of the point source search [19] is such that the diffuse flux cannot be explained as a steady flux from less than around 50 point sources. An IceCube search for neutrinos in association with gammaray bursts [20] strongly constrains models for neutrino emission, and leads to the conclusion that, of the flux in equation 1 , at most $\sim 1 \%$ can come from observable GRBs. Similarly, a search for steady neutrino emission from blazars in the Fermi 2LAC catalogue, while indicating a small excess, limits the contribution from blazars to below 20\% [21].

Neutrinos created in pp interactions will be accompanied by gammas from $\pi^{0}$ decay. These will cascade down in energy and contribute to the extragalactic gamma-ray background (EGB),

\footnotetext{
${ }^{3}$ Each individual astrophysical track event points back at its source, but unless this source is also identified by other means it is not considered here, even if the track is unlikely to be of atmospheric origin
} 
observed by the Fermi Large Area Telescope [22]. Above $100 \mathrm{GeV}$, the EGB can be accounted for by emission from blazars, which are not the dominating contributors to the neutrino flux. It has been pointed out that the contribution from gammas associated with neutrinos from pp interactions, assuming the power law fits to the neutrino spectrum, can saturate or overpredict the EGB [23].

The apparent lack of $\gamma$ emission associated with the neutrinos could be the effect of photohadronic $(\mathrm{p} \gamma)$ interactions dominating the neutrino production. This could suppress the neutrino and gamma fluxes at lower energies and would more naturally give a steeper spectrum than $\gamma \sim 2.2$. Fewer expected low energy neutrino events could also imply less stringent point source limits for contributions from specific source classes. Other suggestions include large contributions from galactic cosmic rays [24].

\section{Conclusion}

IceCube has produced a wealth of physics results; the discussion here concerned the measured astrophysical cosmic ray and neutrino fluxes.

The astrophysical neutrino flux is not understood, and no sources have been identified. Further studies are needed, based on increased statistics, and improved analysis methods. These efforts will benefit from the planned upgrade of the IceCube observatory [25].

\section{References}

[1] P. Kotzer (ed.), Proc. 1975 Summer DUMAND Study, Western Washington State College, Bellingham, WA.

[2] J. G. Learned and S. Pakvasa, Astropart. Phys. 3 (1995) 267.

[3] T. DeYoung, S. Razzaque, and D.F. Cowen, Astropart. Phys. 27 (2007) 283.

[4] Sebastian Böser, "Neutrino mass hierarchy with PINGU", these proceedings.

[5] T. K. Gaisser, T. Stanev, S. Tilav, Front. Phys. China 8 (2013) 748.

[6] IceCube Coll.: M. G. Aartsen et al., Phys. Rev. D88 (2013) 042004.

[7] IceCube Coll.: F. McNally for the collaboration, "Observation of Anisotropy in Cosmic-Ray Arrival Directions with the IceCube Detector", Proc. 2104 Conf. on Ultrahigh Energy Cosmic Rays, Springdale, Utah, 2014; Icecube Coll.: R. Abbasi et al., Astrophys. J. 746 (2012) 33, IceCube Coll.: M. G. Aartsen et al., Astrophys. J. 765 (2013) 55.

[8] G. Giacinti and G. Sigl, Phys. Rev. Lett. 109 (2012) 071101.

[9] A.D. Erlykin and A.W. Wolfendale, Astropart. Phys. 25 (2006) 183; P. Blasi and E. Amato, JCAP 1201 (2012) 011.

[10] IceCube Coll.: M. G. Aartsen et al. Phys. Rev. Lett. 111 (2013) 021103, Phys. Rev. D88 (2013) 112008 .

[11] S. Schönert et al., Phys. Rev. D79 (2009) 043009; T. K. Gaisser et al, Phys. Rev. D90 (2014) 023009.

[12] IceCube Coll.: M. G. Aartsen et al, Science 342 (2013) 1242856.

[13] IceCube Coll.: M. G. Aartsen et al., Phys. Rev. Lett. 113 (2014)101101.

[14] IceCube Coll.: M. G. Aartsen et al., Phys. Rev. D91 (2015) 022001. 
[15] IceCube Coll.: M. G. Aartsen et al. Eur. Phys. J. C75 (2015) 116.

[16] P. Lipari, M. Lusignoli, D. Meloni, Phys. Rev. D75 (2007) 123005; O. Mena, S. Palomares-Ruiz, A. C. Vincent, Phys. Rev. Lett. 113 (2014) 091103; A. Palladino et al., Phys. Rev. Lett. 114 (2015) 171101.

[17] IceCube Coll.: M. G. Aartsen et al., Phys. Rev. Lett. 114 (2015) 171102.

[18] J. K. Becker, Phys. Rep. 458 (2008) 173; U. F. Katz and Ch. Spiering, Prog. Part. Nucl. Phys. 67 (2012) 651.

[19] IceCube Coll.: M. G. Aartsen et al., Astrophys. J. 796 (2014) 109.

[20] IceCube Coll.: M. G. Aartsen et al., Astrophys. J. Lett. 805 (2015) L5.

[21] IceCube Coll.: T. Glüsenkamp for the collaboration, Proc. 5th Roma Int. Conf. on Astroparticle Physics, arXiv:1502.03104.

[22] M. Ajello et al., Astrophys. J. Lett. 800 (2015) L27.

[23] K. Murase, M. Ahlers, and B. C. Lacki, Phys. Rev. D88 (2013) 121301; N. Senno et al., Astrophys. J. 806 (2015) 24; W. Winter, Phys. Rev. D88 (2013) 083007.

[24] A. Neronov and D. Semikoz, arXiv:1412.1690; D. Gaggero et al., arXiv:1504.00227.

[25] IceCube-Gen2 Coll.: M. G. Aartsen et al., arXiv:1412.5106. 